\title{
Corela
}

Cognition, représentation, langage

HS-14 | 2013

Calcul du sens et contexte

\section{De la sémantique pragmatique au contextualisme}

\section{Charlotte Gauvry}

\section{(2) OpenEdition}

\section{Journals}

Édition électronique

URL : http://journals.openedition.org/corela/3483

DOI : $10.4000 /$ corela.3483

ISSN : 1638-573X

\section{Éditeur}

Cercle linguistique du Centre et de l'Ouest - CerLICO

\section{Référence électronique}

Charlotte Gauvry, « De la sémantique pragmatique au contextualisme », Corela [En ligne], HS-14 | 2013, mis en ligne le 25 juin 2014, consulté le 01 mai 2019. URL : http://journals.openedition.org/ corela/3483 ; DOI : 10.4000/corela.3483

Ce document a été généré automatiquement le 1 mai 2019.

\section{(c) (i) (2)(2)}

Corela - cognition, représentation, langage est mis à disposition selon les termes de la licence Creative Commons Attribution - Pas d'Utilisation Commerciale - Partage dans les Mêmes Conditions 4.0 International. 


\title{
De la sémantique pragmatique au contextualisme
}

\author{
Charlotte Gauvry
}

1 Dans l'héritage des travaux inauguraux de Gottlob Frege et du fameux "principe de contexte $»^{1}$ de la préface aux Fondements de l'arithmétique ${ }^{2}$ (« on doit rechercher ce que les mots veulent dire non pas isolément mais pris dans leur contexte [im Satzzusammenhange ]»), la notion de « contexte » a gagné un surcroît d'intérêt dans la philosophie du langage de la seconde moitié du $\mathrm{XX}^{\mathrm{e}}$ siècle, au prix de grandes confusions. Nombreux courants d'analyse d'obédience fort disparate se revendiquent en effet de l'appellation "contextualiste», en partant du principe qu'ils souscrivent au principe de contexte frégéen. Nous entendons ici montrer que derrière ce concept générique de « contexte » se dissimulent une forte polysémie et une hétérogénéité d'usage. Qu'entend-on en effet par «contexte »? Le «contexte propositionnel» de la phrase (comme c'est le cas dans la préface de Frege ou, par exemple, dans la proposition 3.3 du Tractatus logico-philosophicus de Wittgenstein : le Satzzusammenhang en allemand) ou les "circonstances " précises de l'énonciation (die Umstände ou die Umgebung en allemand, the circonstances en anglais)? Indépendamment de la polysémie du concept, il n'est par ailleurs pas acquis que tous s'entendent sur ce que signifie «rechercher ce que les mots veulent dire non pas isolément mais pris dans leur contexte ». Il est tout sauf clair par exemple qu'il soit possible d'associer dans un même élan la stratégie compositionaliste qui refuse de considérer les mots " pris isolément» mais seulement dans leur contexte propositionnel et la « sémantique pragmatique » qui considère que c'est seulement par un recours aux éléments de contexte non sémantiques que l'on peut préciser le sens et, par conséquent (du moins dans sa logique), la valeur de vérité de l'énoncé.

2 Nous proposons ici de tenter de contribuer à la clarification de ces confusions en opérant une distinction entre ce que nous appelons "contextualisme», dans un héritage wittgensteinien et austinien, et « sémantique pragmatique ». La distinction peut sembler ténue mais nous soutiendrons qu'elle est de fait radicale, et pour cette raison même particulièrement difficile à caractériser. Nous soulignerons en effet que la sémantique pragmatique dont l'échelle d'analyse est la proposition, tout en considérant certains 
éléments de contexte extra-sémantique, en reste à une analyse sémantique de la phrase, et donc à l'idée que l'énoncé a un contenu propositionnel susceptible d'être évalué en termes de vérité et de fausseté et que le sens de l'énoncé est déterminé par des « conditions de vérité » (à savoir la manière dont le monde devrait être pour que l'énoncé soit vrai). A contrario, nous tenterons de montrer que le " contextualisme » radical bien compris refuse précisément de s'en tenir à cette approche sémantique de l'énoncé qui considère le sens comme le contenu véri-conditionnel de l'énoncé. Il soutient bien plutôt que le contenu de l'énoncé, si tant est que cela ait même un sens de parler de " contenu ", n'est pas nécessairement propositionnel et que les critères qui en fixent le sens sont euxmêmes déterminés en contexte. C'est dire que le contexte n'intervient pas comme un élément externe qui vient préciser, par apport, dans quelles conditions (ie dans quel état du monde) le sens de la proposition (ou de certains de ses éléments) est vrai ou faux. Il intervient bien plutôt comme la norme qui fixe les critères d'effectuation de l'acte de langage et par là de la compréhension du sens de l'énoncé. Pour le dire dans les termes de l'un des principaux héritiers du contextualisme radical, Charles Travis, c'est la sémantique elle-même qui est "sensible à l'usage»(S-Use sensitivity) ${ }^{3}$. À l'issue de l'analyse de cette distinction, nous espérons jeter une lumière nouvelle sur ce qu'il est convenu d'appeler « contextualisme».

\section{Littéralisme et contextualisme}

3 Pour clarifier notre propos, il nous paraît utile de partir de la typologie des différentes formes de «contextualisme» que propose François Récanati dans son article de 2003 : "Littéralisme et contextualisme : plusieurs variétés $»^{4}$. Si la définition qu'il propose in fine du contextualisme nous paraît contestable, l'article présente le grand mérite de proposer un état des lieux des différentes formes de «contextualisme » qui émaillent la scène analytique contemporaine (en 2003).

4 L'enjeu de l'article est de distinguer deux formes radicales d'analyse du contenu propositionnel d'une phrase, le « littéralisme » et le « contextualisme », et de caractériser la profusion des positions d'analyse intermédiaires. Notons que toutes les différentes positions examinées partagent une hypothèse de base à savoir que l'énoncé possède un « contenu propositionnel ». La question qui oppose le littéralisme au contextualisme est alors de savoir si ce contenu propositionnel s'identifie au contenu sémantique de la phrase, lui-même réglé par les conventions linguistiques de la composition syntaxique. Pour le littéraliste, le contenu de la proposition est le contenu sémantique, alors que pour le contextualiste éliminativiste, le contenu propositionnel est exclusivement déterminé par l'acte de langage. Pour reprendre l'expression dont use Récanati, pour le littéraliste, le contenu propositionnel se détermine "de bas en haut» (bottom-up) et pour le contextualiste, de haut en bas (top-down) : c'est le recours à des éléments non sémantiques (pragmatiques) qui le détermine.

5 Dans l'article, Récanati commence par distinguer quatre versions du littéralisme: le " proto-littéralisme » considère que le contenu propositionnel est entièrement déterminé par le contenu sémantique de la phrase et que les phénomènes de dépendance contextuelle sont un défaut intrinsèque des langues vernaculaires à combattre; l'« éternalisme » concède quant à lui l'existence de termes dont le contenu propositionnel requiert une détermination contextuelle (les indexicaux) mais il considère ces phénomènes comme subalternes et éliminables; le "conventionnalisme» refuse de 
considérer que ces phénomènes d'indexicalité sont éliminables mais maintient que, en dernière instance, ce sont les conventions linguistiques sémantiques, et non pas les « intentions du locuteur », qui fixent le contenu des indexicaux en respect du contexte. La dernière version du littéralisme (la plus courante) est appelée «minimalisme ». Elle ne tolère que les variations contextuelles appelées par les conventions linguistiques : ce n'est qu'à la condition que la phrase elle-même le requière qu'une influence contextuelle peut être considérée.

6 Entre le littéralisme (dont on vient d'énumérer succinctement quatre versions et que l'article analyse bien plus en détail) et le contextualisme, Récanati introduit deux positions intermédiaires: la conception syncrétique et le "quasi-contextualisme ». La conception syncrétique se présente comme un compromis. Elle admet deux niveaux de contenu : le contenu correspondant à la phrase, celui de la proposition «minimale », et le contenu de l'acte de langage qui est le contenu intuitif qu'on accorde à l'énoncé. Récanati prend pour l'illustrer un exemple de Scott Soames ${ }^{5}$. Quand on demande dans un bar : « Un café, s'il vous plaît. ", le contenu sémantique de la phrase ne donne aucune indication sur ce qui est effectivement demandé: si "café» indique une fève ou le café moulu par exemple, ni si le café est attendu dans une tasse, un sac, un baril, etc. Cette position consiste à distinguer le contenu sémantique d'une phrase (le dénominateur commun minimal à tous les usages d'une phrase) de ce qui est communiqué par l'usage de la phrase (qui, en contexte, contribue à déterminer le dénominateur commun). Récanati distingue cette première position intermédiaire du «quasi-contextualisme» qui abandonne l'idée de contenu sémantique et se réfère à des expressions dont on pourrait moduler le contenu en fonction du contexte. Récanati prend l'exemple de la phrase : «le lion est dans la cour ». En contexte, on comprend que par «lion », c'est à la statue d'un lion qu'il est fait référence. La position est décrite comme seulement "quasi» contextualiste car elle n'est pas éliminativiste: elle définit le contenu minimal comme inutile mais non comme dépourvu de sens.

7 Enfin, par contextualisme (authentique), Récanati caractérise en conséquence trois positions qu'il nomme respectivement le « principe de composition pragmatique » (PC), le "principe de mauvais format» (Wrong Format View ou WF) et l'éliminativisme sémantique. La première position (PC) défend que le sens est littéral et que les modulations contextuelles sont optionnelles. Mais elle adopte un processus de composition pragmatique. Même si elle défend que le contenu propositionnel d'un mot isolé est fixé par la sémantique (et par le contexte dans le cas des expressions indexicales), elle soutient que la détermination du contenu de la phrase requiert un recours au contexte non sémantique ${ }^{6}$. Récanati développe, entre autres, un exemple éclairant pour préciser cette position : un dialogue du film Desire de Franck Borsage de 1936. Au personnage « Pedro », un autre personnage demande : « Apporte l'assiette dans la cuisine et désarme le fricassé ! ». Pour déterminer le contenu propositionnel de «désarmer le fricassé », le contenu sémantique ne suffit pas. Même en sachant ce que veut dire "désarmer » et « fricassé » et à quel état du monde correspond l'énoncé, on ne peut pas y parvenir. Seul un recours au contexte peut expliquer la composition inhabituelle, à savoir que Gary Cooper (l'acteur) tend une assiette au serveur Pedro qui contient un fricassé et un pistolet. Il faut aussi savoir qu'un combat vient d'avoir lieu. Par le recours au contexte, on comprend alors quel «contenu propositionnel» accorder à «désarmer le fricassé »" Selon cette position, les mots ont donc un sens littéral abstrait mais ils n'ont de sens (à 
savoir de valeur de vérité) qu'au sein d'une phrase dont la composition est déterminée en contexte.

Récanati oppose ce premier type de contextualisme à un deuxième plus radical: «le principe de mauvais format ». Selon ce deuxième principe, ce n'est pas seulement la composition pragmatique qui requiert un éclairage du contexte. Les mots individuels euxmêmes ne peuvent pas être "interprétés ${ }^{8}$ directement car ils n'ont pas le "bon format » pour cela. Leur sens est trop abstrait, ou trop riche, pour pouvoir être interprété en dehors du processus de la construction du sens dans le contexte de la proposition.

Enfin, Récanati clôt sa typologie par la forme de contextualisme qui nous intéresse: le " contextualisme radical éliminativiste ", présenté comme le contextualisme d'Austin et de Wittgenstein. Cette version éliminativiste déclare faire l'économie de la sémantique. Elle soutient que le contenu propositionnel ne se détermine que par son application en contexte, indépendamment de toute règle sémantique. Ce sont les usages précédents de la phrase qui tiennent lieu d'«apport» au contenu propositionnel. En fonction d'une situation de référence (target situation) ou « situation-source », en contexte, l'évaluation du contexte propositionnel se détermine par variation". Selon Récanati, les innombrables exemples de Charles Travis procurent une illustration de ce " phénomène de modification vériconditionnelle » sur fond de « contraste $»^{10}$.

10 C'est cette conclusion qui nous intéresse. Il nous semble que par la méthode adoptée, Récanati nous délivre un état des lieux des différentes méthodes d'analyse du contenu propositionnel d'un énoncé et à ce titre ne sort pas de la sémantique. Le but de l'article de 2003 est en effet de montrer comment le «proto-littéralisme» originaire s'est progressivement rapproché du contextualisme, par divers ajustements progressifs, pour parer à ses écueils. Ainsi présenté, le contextualisme se présente comme le dernier maillon de la stratégie d'adaptation du littéralisme. C'est précisément là, selon nous, le péché originel de la typologie de Récanati. Car on ne peut espérer saisir le contextualisme dans sa radicalité en le considérant comme une modulation (par complément) du littéralisme sémantique. Nous entendons bien plutôt montrer ici que la rupture est plus radicale : le contextualisme n'est pas un ajustement du littéralisme mais une remise en cause de ses fondements. Par une stratégie de contrastes, qui opère par modulations ${ }^{11}$ et ajustements, on ne peut pas parvenir à saisir le "contextualisme» dans sa radicalité. Pour le dire autrement, tel qu'il est présenté dans l'article, "le contextualisme éliminativiste » reste un contextualisme sémantique. A contrario, le « contextualisme » qui nous intéresse rompt, à son fondement, avec l'illusion descriptiviste ${ }^{12}$ qui consiste à attribuer à un énoncé un contenu propositionnel déterminé par des conditions de vérité. L'enjeu du contextualisme conséquent (et a fortiori des exemples de Charles Travis) est moins de souligner les modulations du contenu propositionnel selon sa coloration contextuelle que de rompre avec tout modèle qui attribue un contenu vériconditionnel à la proposition.

\section{De la « sémantique pragmatique » au contextualisme}

11 Pour distinguer le contextualisme radical du «contextualisme sémantique » ou de la "sémantique pragmatique », on propose de continuer à prendre appui sur les travaux récents de Récanati qui nous semblent remarquablement clairs et paradigmatiques de 
cette stratégie d'assimilation. On s'appuie sur son dernier livre paru en français en 2008, Philosophie du langage (et de l'esprit) ${ }^{13}$. Le livre présente l'intérêt de préciser ce que l'on peut qualifier de "sémantique pragmatique ", en se revendiquant, de manière problématique, de l'héritage de Wittgenstein.

La préface du livre annonce clairement le programme. Récanati commence par dresser un parallèle entre "langage» et "pensée» (justifiant le titre de l'ouvrage) ${ }^{14}$. En conséquence, il isole trois propriétés communes au langage et à la pensée: la "structure », la "syntaxe » et la "sémantique». Si cette typologie est contestable, elle isole, de manière instructive, trois « propriétés » dites paradigmatiques du langage et de la pensée. C'est la dernière qui nous intéresse ici : la « sémantique ». Récanati en propose une définition dans son glossaire : «la sémantique est la discipline qui s'occupe du sens (du contenu) des formes linguistiques $»^{15}$. On comprend que le sens est défini comme un élément de contenu interne à la phrase. Or, c'est dans ce cadre structurel (sémantique) et selon cette modalité (en tant que contenu) que la notion de «contexte» est ensuite introduite.

Dans un mouvement résolument polémique, pour contrer les apories d'une sémantique dite "référentielle», Récanati entend faire droit au caractère non pas seulement sémantique de la phrase mais à la valeur d'acte de l'énoncé. Une telle revendication s'inscrit, de manière contestable, dans l'héritage conjoint de Grice, Austin et Wittgenstein ${ }^{16}$ :

Il y a donc deux dimensions distinctes - le contenu et la fonction - et la question qui se pose est celle de savoir ce qui prime. Une sémantique pragmatique, distincte à la fois d'une sémantique référentielle et d'une sémantique cognitive, considère l'emploi, la fonction, comme l'élément fondamental: donner le sens d'une expression, c'est dire quel est son emploi, à quoi elle sert. Suivant le slogan attribué à Wittgenstein, «le sens, c'est l'emploi », [meaning is use]. ${ }^{17}$

On ne peut manquer d'être surpris par la référence à Wittgenstein. S'il est vrai que, d'un point de vue wittgensteinien (du moins celui des Recherches), on doit récuser la pertinence d'une « sémantique référentielle » « qui associe aux mots des choses (leur dénotation) » et d'une " sémantique cognitive » "qui associe au mot des représentations mentales $»^{18}$, il nous semble tout aussi problématique de qualifier la méthode grammaticale wittgensteinienne de "sémantique pragmatique ». Il est juste que Wittgenstein accorde une importance, dès le Tractatus logico-philosophicus puis dans les Recherches philosophiques, à l'usage qui est fait d'un signe au sein d'une proposition puis d'un jeu de langage. Mais le réglage de cet usage n'est précisément pas sémantique. Il n'est plus question de " sémantique » (du moins au sens de Récanati) pour Wittgenstein, ni même à strictement parler de « pragmatique $»^{19}$. Pour Récanati, c'est en revanche en tant que détermination du contenu vériconditionnel de l'énoncé que le «contexte » est introduit, au sein de la phrase. C'est manifeste dans sa définition de l'indexicalité.

Rappelons que Récanati hérite du concept d'indexicalité par les travaux de David Kaplan et qu'il s'inscrit dans la filiation des analyses de Peter Strawson, Gareth Evans et John Perry ${ }^{20}$. Du célèbre article de Kaplan, «Demonstratives » ${ }^{21}$, il retient la distinction entre le « contenu » et le « caractère » de l'indexical. L'indexical a une sémantique définie par le caractère qui est une règle linguistique et qui lui permet de déterminer un contenu sémantique en fonction du contexte. C'est une fonction de contexte à contenu - le contenu en question étant le contenu sémantique de l'énoncé. Récanati précise cet héritage dans son glossaire, point l) «Indexicalité : caractère et contenu » : 
Les expressions indexicales comme «je ", «tu», « ici », " maintenant » ont une double propriété. D'une part, elles ne font référence que relativement au contexte d'énonciation. «Je» désigne le locuteur, «tu» l'interlocuteur, «ici » le lieu de l'énonciation, etc. D'autre part, elles sont directement référentielles: elles introduisent un individu (le référent) dans la condition de vérité ou la proposition exprimée ${ }^{22}$. définies comme «directement référentielles ». C'est leur "référent » qui détermine le sens de l'indexical et, en conséquence, selon une acception vériconditionnelle du sens, la « condition de vérité » de l'énoncé. Le « caractère » de l'indexical - qui l'oriente (soit « la propriété d'être le locuteur, ou l'interlocuteur, ou le lieu, ou le moment de l'énonciation) - n'intervient pas comme condition de vérité de l'énoncé. Il guide l'interprétation de la phrase et indique comment elle doit compléter le contenu sémantique de l'énoncé : «il sert à déterminer le référent en contexte ». Ainsi entendu, l'indexical a une sémantique, orientée par un "caractère", qui lui permet de déterminer, en contexte, un contenu. Récanati prolonge l'analyse en précisant que les "indexicaux ont une fonction spécifique » : «ils servent à emmagasiner les informations obtenues en vertu d'une certaine relation au référent $»^{23}$. Ce sont des « dossiers mentaux ».

Dans une telle perspective, le contexte est un élément de contenu de la sémantique. Il est défini comme l'arrière-plan d'information susceptible de compléter le sens des «constituants» en fournissant un complément de contenu. Aussi, loin d'ouvrir la sémantique à la pragmatique, Récanati rabat insidieusement la pragmatique sur la sémantique. Il reconduit les éléments non-sémantiques à des éléments que la phrase est susceptible d'absorber. C'est particulièrement manifeste dans la manière dont Récanati reconduit « le contenu de l'acte de parole » au « contenu représentationnel de la phrase » qui le « reflète » :

$\mathrm{Au}$ lieu d'opposer le contenu représentationnel de la phrase et les indications qu'elle véhicule concernant l'acte de parole accompli par son énonciation, on peut considérer que le contenu représentationnel de la phrase ne fait lui-même que refléter le contenu de l'acte de parole dont elle est l'instrument ${ }^{24}$.

En définissant l'acte de parole par son " contenu » vériconditionnel, lui-même reconduit au contenu propositionnel qu'il vient compléter, il semble que la sémantique pragmatique succombe à l'illusion descriptiviste et absorbe le contexte dans la sémantique. Elle ouvre la brèche vers un anticontextualisme radical, plus menaçant encore que le littéralisme, qui conduit non pas à délaisser le contexte mais à le dissoudre.

\section{Le contextualisme radical}

Il convient alors de distinguer la « sémantique pragmatique » du contextualisme qui nous intéresse. À bien des égards, comme nous l'avons montré, la " sémantique pragmatique ", malgré ses efforts pour rompre avec la sémantique dite "référentielle", se présente comme un dernier reliquat du « descriptivisme » que dénonce Austin dans Quand dire c'est faire. Car dénoncer "l'illusion descriptiviste", à la suite d'Austin, enjoint à renoncer à tout traitement sémantique des actes de langage et non à compléter (même intégralement) la sémantique par des éléments de contexte. L'anti-descriptivisme conventionnaliste d'Austin intime l'exigence incompressible de renoncer à la notion de "contenu propositionnel vériconditionnel ", aussi bien au niveau de l'analyse du locutoire que de l'illocutoire. En conséquence, aussi paradoxal que cela puisse paraître,

Corela, HS-14 | 2013 
d'un point de vue contextualiste, le littéralisme est plus conséquent que la « sémantique pragmatique » quand il asserte que le contenu propositionnel de l'énoncé n'est jamais que son contenu sémantique. Car le contextualisme conséquent, pour rompre avec la sémantique, ne doit pas ajuster un «contenu propositionnel» à la dimension pragmatique du langage, ni même considérer que le «contenu» de l'énoncé est entièrement déterminé par l'usage qui en est fait, mais rompre avec toute approche vériconditionnelle du langage en termes de "contenu». Aussi, il faut adresser à la sémantique pragmatique les critiques que l'on a pu traditionnellement adresser à Grice et à sa reformulation du conventionnalisme austinien. Ce reproche n'est pas fortuit dans la mesure où Récanati, sans toujours en prendre lui-même l'exacte mesure, s'inscrit bien plus directement dans l'héritage de Grice que de celui d'Austin, tout en mésestimant, nous semble-t-il, les différences fondamentales entre les deux auteurs.

Tentons par conséquent de préciser plus positivement ce que nous appelons « contextualisme ». Une première précision s'impose. Dire que le sens de l'énoncé n'est pas reconductible à une valeur de vérité ( "vrai » ou « faux ») conditionnée par un état du monde ne signifie évidemment pas que la perspective contextualiste fait l'économie de l'exigence de correction et adopte une position relativiste selon laquelle cela n'aurait pas de sens de décréter qu'un énoncé est plus ou moins correct. Bien au contraire, le contextualisme, tel qu'il s'énonce par excellence chez Austin, est conventionaliste. Il défend que tout acte de langage est une pratique normée et qu'il est donc, par définition, toujours plus ou moins bien réussi. Mais l'enjeu du contextualisme est précisément de montrer que c'est le "contexte " lui-même qui détermine les normes en question. Le contexte se présente donc comme les circonstances conventionnellement déterminées dans lesquelles on s'attend, normalement, à telles ou telles pratiques discursives déterminées. Pour reprendre les exemples très connus d'Austin de pratiques institutionnalisées : dans le contexte du baptême du bateau, on s'attend à un acte de langage du type «je baptise ce bateau le Queen Mary... »; dans celui du mariage, on attend le « oui » qui scelle l'union, etc. Mais l'enjeu des analyses d'Austin et des exemples de Charles Travis que cite Récanati est de montrer que tous nos actes de langage ordinaires s'inscrivent dans de tels contextes déterminants. Pour reprendre les exemples, également bien connus, de Charles Travis ${ }^{25}$, c'est en contexte que l'on fixe le sens d'un énoncé comme : «Il y a du lait dans le frigo.». Pour un même fait (un réfrigérateur dans lequel un peu de lait a coulé dans le bac du fond), l'énoncé sera correct dans certains contextes (Odile vérifie si Hugo a correctement nettoyé le frigo et remarque, à juste titre, d'un ton réprobateur, qu'il reste du lait dans le frigo). Dans un autre contexte (Hugo n'a pas terminé son petit déjeuner et a encore faim), le même énoncé sera incorrect. C'est seulement en ce sens que le contextualisme défend la thèse que le sens ne se détermine et n'est correct ou incorrect qu'en contexte. On cite Charles Travis : «c'est seulement dans des circonstances appropriées que les mots ont du sens ; c'est seulement chez eux ou dans un environnement [surroundings] approprié qu'ils expriment une pensée dont le contenu est suffisamment défini pour autoriser, entre autres choses, une évaluation en termes de vérité $»^{26}$.

21 Une telle affirmation peut sembler recouper la thèse de la sémantique pragmatique selon laquelle le contenu propositionnel de l'énoncé (défini comme son sens) ne se détermine que dans le contexte pragmatique de l'énonciation. Mais ce serait là négliger un déplacement considérable, à savoir que le contextualisme entend précisément récuser la notion de contenu véri-conditionnel, c'est-à-dire l'idée que la vérité de l'énoncé est 
conditionnée par un état de chose (quand bien même cet état de chose serait précisé par des éléments de contexte). On peut même aller jusqu'à dire que l'analyse sémantique de l'énoncé présente un tour anti-contextualiste dans la mesure où elle récuse son principe constitutif, à savoir que le contexte n'est pas un élément de contenu (ni même ce qui détermine intégralement le contenu de l'énoncé) qui précise les conditions de vérité de l'énoncé mais la norme qui détermine le standard de correction de l'effectuation de l'acte de langage. Aussi, le rôle du contexte est à ce point fondamental que, pour le contextualiste, il n'y a d'actes de langage qu'en contexte. Ce n'est pas seulement la valeur de vérité des énoncés, ni même son sens (défini comme " contenu ») qui sont définis en contexte, mais bien le standard de correction qui définit un acte de langage comme acte.

En soulignant cette différence, on espère d'abord avoir suscité une méfiance à l'égard de l'usage qui est fait du concept de «contexte». Indépendamment de la polysémie du concept, il est clair que par "contexte» et "contextualisme", on peut indiquer des méthodes d'analyse de l'énoncé et de son sens fort différentes. C'est alors la nature du contextualisme que nous défendons, contextualisme radical, que nous espérons avoir précisée. Nous avons tenté de montrer que le contextualisme appelle non pas à compléter la sémantique, ni même à la dépasser, mais bien plutôt à la critiquer à son fondement. À ce titre, le " contextualisme sémantique » ou la " sémantique pragmatique » présente une contradiction dans les termes mêmes de leur formulation.

\section{NOTES}

1. Sur cette question, nous renvoyons aux travaux de Jocelyn Benoist, notamment au chapitre VIII de Sens et sensiblité, Paris, Cerf, 2009 et à ses deux introductions aux textes de Frege, "Sur le sens et la référence " et "La pensée », publiés dans le recueil Philosophie du langage. I. Signification, vérité et réalité, B. Ambroise et S. Laugier (éd.), Paris, Vrin, 2009. Nous renvoyons également à notre article publié dans la revue en ligne Implications philosophiques: "Principe de contexte et circonstances. De Frege à Wittgenstein » dans le dossier "Wittgenstein en dialogues » de 2011.

2. G. Frege, Die Grundlagen der Arithmetik: eine logisch mathematische Untersuchung über den Begriff der Zahl, Hamburg, Meiner, 1986 ; trad. fr. par Cl. Imbert, Les Fondements de l'arithmétique : recherche logico-mathématique sur le concept de nombre, Paris, Seuil, 1969.

3. Voir Ch. Travis, The Uses of Sense. Wittgenstein's Philosophy of Language, Oxford et New-York, Oxford University Press, $1989,2001^{2}$, p. 18 sqq. et plus précisément le chapitre 4 de la première partie.

4. F. Récanati, "Literalism and Contextualism: Some Varieties", Contextualism, Oxford, Oxford University Press, 2003. On trouve l'article en ligne. (L\&C par la suite). Nous remercions Vincent Richard et Bruno Ambroise de nous avoir indiqué l'existence de cet article. Nous proposons notre traduction.

5. S. Soames, Beyond Rigidity: The Unfinished Semantic Agenda of Naming and Necessity, New-York, Oxford University Press, 2002, p. 78.

6. Voir L\&C, p. 14. 
7. Récanati cite plusieurs exemples bien connus de Searle. Il cite notamment l'exemple de l'article «The Background of Meaning » du verbe « couper». Si le sens sémantique de " couper » est fixé, il reste que dans des compositions différentes, par exemple dans "Bill coupe le gazon » ou Sally coupe le gâteau ", " couper " n'a pas le même sens. On ne coupe pas le gazon avec un couteau. Ainsi que le souligne Récanati (LC, p. 11), l'argumentation de Searle repose sur l'idée que la composition s'inscrit dans un "arrière-plan d'assomptions ». Inversement, dans une phrase dépourvue d'un tel arrière-plan comme « couper le soleil », on ne peut pas déterminer le sens de " couper ». Voir J. Searle, "The Background of Meaning " in Speech Act Theory and Pragmatics, J. Searle, F. Kiefer et M. Bierwisch, Dordrecht, Reidel, 1980, pp. 222-223. Voir aussi J. Searle, «Literal Meanings », Erkenntnis, 12, 1978, p. 207-224.

8. L\&C, p. 18

9. L\&C, p. 22 .

10. L\&C, p. 23.

11. Par enrichissement, transfert de prédicat ou atténuation.

12. Par «illusion descriptiviste ", Austin critique un conception représentationaliste du langage qui ne parvient pas à comprendre que le langage ne sert pas à représenter le monde mais à accomplir un certain nombre d'actes. Voir J. Austin, How To Do Things With Words, Cambridge (Mass.), Harvard University Press, 1962, p. 3., trad. fr. par G. Lane, Quand dire, c'est faire, Paris, Seuil, 1970, p. 39. Mais la critique, authentiquement comprise, vise aussi toute forme de descriptivisme insidieux qui en reste à une analyse sémantique des actes de langage et qui reconduit l'analyse du langage à celle de la phrase.

13. F. Récanati, Philosophie du langage (et de l'esprit), Paris, Gallimard, 2008.

14. Ceci pour justifier le constat empirique que la plupart des dits philosophes du langage des années 1970 sont devenus des philosophes de l'esprit dans les années 1980-1990.

15. F. Récanati, Philosophie du langage (et de l'esprit), op. cit. p. 246.

16. Voir notamment le chapitre 5 de Philosophie du langage (et de l'esprit), «Langage et acte de parole ", pp. 101-117. Selon nous, la faute originelle de Récanati consiste à identifier la lecture du contexte de Wittgenstein et d'Austin à celle de Grice, ou du moins à sous-estimer le déplacement considérable opéré par Grice. Car Grice, en considérant que le sens d'une phrase peut être déterminé par désambiguation (syntaxique et lexicale) et par l'attribution de valeur aux indexicaux, en reste à une conception sémantique de la phrase. Par ailleurs, comme le notent $\mathrm{B}$. Ambroise et S. Laugier dans l'introduction au volume II des Textes-clé de Philosophie du langage : « le projet de Grice, explicitement anti-austinien, est d'offrir une explication fondationnelle de la signification non-naturelle en termes d'intentions du locuteur (plutôt qu'en termes de conventions, qui interviennent secondairement) », Philosophie du langage. II. Sens, usage et contexte, B. Ambroise et S. Laugier (éd.), Paris, Vrin, 2011, p. 27.

17. F. Récanati, Philosophie du langage (et de l'esprit), op. cit., p. 86.

18. Pour les deux citations précédentes, ibid, p. 85.

19. Précisément car Wittgenstein rompt avec la logique des modèles d'analyse qu'adopteront à sa suite Grice et Searle en considérant le contexte (éventuellement implicite) comme un élément de détermination de la sémantique. Voir par exemple, S. Laugier, « Acte de langage ou pragmatique ?», Revue de métaphysique et de morale, 2004/2, Paris, PUF, pp. 279-303.

20. Voir F. Récanati, Philosophie du langage, op. cit., p. 202. Il faudrait cependant distinguer ces trois influences.

21. D. Kaplan, "Demonstratives ", in Themes from Kaplan, J. Almog, J. Perry, H. Wettstein (éd.), New-York et Oxford, Oxford University Press, 1989.

22. F. Récanati, Philosophie du langage, op. cit., pp. 255-256.

23. Ibid, p. 202.

24. Ibid, p. 97. 
25. Et que nous avons travaillés récemment dans notre article "L'interprétation du sens des énoncés. Une lecture contextualiste ", revue en ligne Methodos. Savoirs et textes, dossier " Interpréter en contexte », Ch. Berner (dir.), volume 13, 2013.

26. Ch. Travis, The Uses of Sense, op. cit., p. 36.

\section{RÉSUMÉS}

Dans l'héritage des travaux inauguraux de Gottlob Frege et du fameux « principe de contexte » de la préface aux Fondements de l'arithmétique, la notion de " contexte » a gagné un surcroît d'intérêt dans la philosophie du langage de la seconde moitié du XX $\mathrm{XX}^{\mathrm{e}}$ siècle, au prix de grandes confusions. Notre article tente de contribuer à la clarification de ces confusions en proposant une distinction entre le « contextualisme », tel qu'il se définit dans un héritage wittgensteinien et austinien, et la "sémantique pragmatique ». Nous espérons ainsi montrer que la sémantique pragmatique dont l'échelle d'analyse est la proposition, tout en considérant certains éléments de contexte extrasémantique, en reste à l'idée que l'énoncé a un contenu propositionnel susceptible d'être évalué en termes de vérité et de fausseté et que le sens de l'énoncé est déterminé par des « conditions de vérité » (à savoir la manière dont le monde devrait être pour que l'énoncé soit vrai) : elle en reste donc à une analyse sémantique de la phrase. A contrario, nous tenterons de montrer que le «contextualisme radical» bien compris refuse précisément de s'en tenir à cette approche sémantique de l'énoncé et définit le contexte non pas comme un élément de «contenu » de la phrase mais comme la norme qui détermine un acte de langage en tant qu'acte.

Following Gottlob Frege's seminal work, in particular the famous « context principle » from Die Grundlagen der Arithmetik's foreword, the notion of «context " gained prominence in the philosophy of language during the second half of the 20th century. This generated however major misunderstandings. This paper seeks to partly clarify those misunderstandings by introducing a distinction between "contextualism" (from a wittgensteinian and austinian point of view) and "pragmatical semantics". It intends to show that pragmatical semantics, whose analyses focus exclusively on the proposition, while taking into account some extra-semantics pieces of context, maintains the idea that an utterance has a propositional content which can be evaluated as "true" or "false" and that the sense of an utterance is determined by truth conditions (that is the way the world should be for an utterance to be true). In this sense, pragmatical semantics remain a semantical analysis of the sentence. The paper seeks to show that, on the opposite, the right understanding of "radical contextualism" refuses precisely this semantics approach of the utterance and defines the context not as a piece of "content" in a sentence but as the norm which determines a speech act as an act.

\section{INDEX}

Mots-clés : sens, sémantique, contextualisme, littéralisme, composition, proposition, langage, usage

Keywords : meaning, semantics, contextualism, literalism, composition, proposition, language, usage 
AUTEUR

CHARLOTTE GAUVRY

Université Albert Ludwig de Fribourg-en-Brisgau, Allemagne 